\title{
Artículo
}

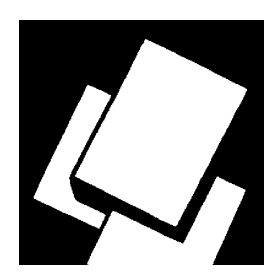

\section{Burnout e intenciones de jubilación anticipada entre empleados mayores*}

\section{Burnout and early retirement intentions among older employees}

\author{
KÈNE HENKENS 1 \\ MONIQUE LEENDERS ${ }^{1-2}$
}

\section{RESUMEN}

El tema central de este artículo es la jubilación y el burnout de los empleados mayores. Analizamos si existe una relación entre las dimensiones de agotamiento emocional y despersonalización características del burnout y las intenciones de jubilación de los empleados mayores. Asimismo, se investiga si existe una relación entre las intenciones de jubilación y las características del puesto de trabajo, el apoyo social y la autoeficacia, así como con las citadas dimensiones del burnout. Los datos proceden de una investigación con empleados holandeses y sus parejas ( $\mathrm{N}=2.892)$. Los resultados permiten ver que la carga laboral excesiva, un trabajo duro desde el punto de vista físico y una ausencia demasiado grande de desafíos están relacionados con los sentimientos de agotamiento emocional. La despersonalización se explica por la escasez de desafíos en el trabajo, la carga laboral excesiva, una falta de apoyo social por parte de los compañeros y una baja autoeficacia. El agotamiento emocional, la despersonalización y las relaciones conyugales explican en gran medida las intenciones de jubilación. El burnout y el proceso de jubilación están muy relacionados, pero parecen ser hasta cierto punto dos procesos diferentes. Mientras que el burnout se determina esencialmente dentro del entorno laboral, los factores externos al trabajo son cruciales en la comprensión de la jubilación.

\footnotetext{
${ }^{1}$ Nederlands Interdisciplinair Demografish Institut (NIDI).

2 Radbouduniversiteit (Departamento de Psicología del Trabajo y las Organizaciones).

* Esta investigación se llevó a cabo en el marco del proyecto NWO-VIDI: "The process of retirement: A dynamic and multi-actor perspective" [El proceso de jubilación. Una perspectiva dinámica y multiactoral].
}

Traducción del neerlandés al castellano realizada por Marta Malo de Molina. 


\begin{abstract}
The central subject of this article is retirement and burnout among older workers. We explored whether there is a relationship between burnout dimensions exhaustion and despersonalization and retirement intentions. We also studied the relationship between retirement intentions and job characteristics, social support and self-efficacy as well as the dimensions of burnout. The data were taken from a survey administered to Dutch older workers and their spouses $(\mathrm{N}=2,892)$. The results show that heavy workload, a physically taxing work, and lack of challenge explain exhaustion. Despersonalization is explained by lack of challenge, heavy workload, lack of peer support, and self-efficacy. Exhaustion, despersonalization and marital interactions explain most retirement intentions. Although burnout and retirement are related, they appear to involve different processes. While burnout can generally be explained by work environment, non-work related factors enhance our understanding of retirement intentions.
\end{abstract}

\title{
PALABRAS CLAVE
}

Burnout, Jubilación anticipada, Intenciones de jubilación, Empleados mayores.

\section{KEY WORDS}

Burnout, Early retirement, Retirement intentions, Older employees. 


\section{INTRODUCCIÓN}

Para hacer frente a los costes del envejecimiento y garantizar un potencial laboral suficiente, el aumento de la participación laboral de los empleados mayores constituye una punta de lanza de la actual política gubernamental en Holanda ?véase el informe reciente de la comisión Bakker (Bakker Comisión Report, 2008). Para fomentar la participación laboral de este grupo, se han adoptado en los últimos años diferentes medidas, estipuladas en la Ley de jubilación anticipada, pensión de prejubilación y ahorro laboral [Wet vervroegd uittreden, prepensioen en levensloopregeling (VPL) $]^{1}$, que entró en vigor el 1 de enero de 2006. Estas medidas incluyen, entre otras cosas, que las normas de la jubilación anticipada [Vervroegde Uittreding (VUT)], la pensión de prejubilación y la pensión puente se hagan poco atractivas desde el punto de vista económico. Por otro lado, está la posibilidad de que los empleados elijan su propio porcentaje de ahorro laboral [levensloopregeling], lo cual puede utilizarse para dejar de trabajar antes (Wapperom, Boskemper y Schouten, 2006).

Hemos dejado atrás la época en que la jubilación suponía un cambio brusco de una vida de trabajo a una vida de ocio. Resulta más frecuente que antes que las personas se mantengan activas de una manera $u$ otra en el mercado de trabajo después de la jubilación. No obstante, hay también indicios de que, en ocasiones, el proceso de jubilación se empieza mucho antes de que se haya alcanzado la edad de jubilación. El momento de dejar de trabajar constituirá en estos casos la pieza final de una retirada mental paulatina de la relación laboral iniciada años antes. Los directivos dicen que las personas que se colocan en el carril de la jubilación ya están mentalmente jubiladas o desenganchadas. Hanisch y Hulin (1990) estiman que cabe considerar la jubilación anticipada como una forma de retirarse de la organización para evitar situaciones desagradables en el puesto de trabajo y que hay que comparar esta actitud con otras formas de retirada del contexto laboral, como el absentismo y el distanciamiento psicológico. De la investigación holandesa de Henkens y Van Solinge (2003) se desprende que el $8 \%$ de los empleados toman distancia "mental" del trabajo mucho antes de la edad legal de jubilación y que este distanciamiento psicológico de la situación laboral se acentúa a medida que se acercan a la edad legal de jubilación. Todavía se sabe muy poco sobre los factores determinantes de esta conducta de preencarrilamiento y distanciamiento y sobre la relación entre estos procesos y las intenciones de jubilación.

Este artículo se centra en una forma especial de distanciamiento mental del proceso laboral y de caída de la productividad, a saber, el burnout. De acuerdo con la definición de Maslach y Jackson (1981), el burnout es un síndrome caracterizado por agotamiento emocional, cinismo y sentimiento de ineficacia y falta de competencia. El burnout está relacionado con una merma de la productividad, un mayor absentismo laboral y la intención de dejar

\footnotetext{
${ }^{1}$ El levensloopregeling o ahorro laboral, también conocido simplemente como levensloop, es una normativa fiscal aprobada en Holanda el 1 de enero de 2006 para facilitar el ahorro de cara a generar una renta sustitutiva para periodos de excedencia o baja no remunerada, por ejemplo, antes de la jubilación. El empresario está obligado a colaborar, mientras que el empleado puede decidir qué porcentaje de su salario debe reservarse para este ahorro o levensloop y en qué organismo se depositará [N. de la T.].
} 
Burnout e intenciones de jubilación anticipada entre empleados mayores

el empleo (Kickul y Posig, 2001; Maslach y Jackson, 1981; Pines y Aronson, 1988; Maslach, Schaufeli y Leiter, 2001).

Apenas hay investigaciones sobre los factores determinantes del burnout entre empleados mayores. Esta laguna llama la atención cuando se supone que el aumento del burnout está ligado a los cambios constantes en el trabajo y en el entorno de trabajo (Kickul y Posig, 2001; Maslach, 1998; Shirom, 2003) y a los mayores se les asocia precisamente con la imagen estereotípica de que les cuesta sobrellevar las exigencias organizativas cambiantes (Visser, Henkens y Schippers, 2003).

En este artículo abordaremos en primer lugar la influencia del entorno de trabajo, el apoyo social y la personalidad (autoeficacia) sobre dos dimensiones importantes del burnout, a saber, el agotamiento emocional y la despersonalización. A continuación, nos ocuparemos de la relación entre burnout e intenciones de jubilación de los empleados mayores. La investigación de la relación entre burnout y jubilación puede mejorar nuestra comprensión del carácter procesual de la jubilación, donde el momento de retiro puede venir precedido por una merma de la productividad del trabajo a causa del burnout.

En el análisis del proceso de jubilación, se han realizado muchas investigaciones sobre los factores que inciden en las intenciones de jubilación de los empleadores mayores (Adams, 1999; Beehr, Glazer, Nielson, y Farmer, 2000; Henkens y Van Solinge, 2003; Henkens y Tazelaar, 1994). De diferentes investigaciones se desprende que las decisiones de jubilarse están influidas por la situación económicofinanciera, la salud, características del trabajo, como el trabajo monótono y apenas desafiante (Henkens y Tazelaar, 1997), las posibilidades de hacer carrera (Adams, 1999; Koloski, Ekerdt y DeViney, 2001), la autonomía (Beehr et al., 2000) y la relación con la pareja (Davey y Szinovacz, 2004; Henkens y Van Solinge, 2002; Henkens y Tazelaar, 1994; Smith y Moen, 1998). El debilitamiento de la salud constituye uno de los factores determinantes más importantes de la jubilación anticipada y es evidente que los empleados mayores tienen una intención más firme de abandonar el mercado de trabajo de manera prematura a medida que va mermando su salud.

La mayor parte de la investigación sobre la jubilación anticipada se centra, no obstante, en la salud general (Adams, 1999; Adams, Prescher, Beehr y Lepisto, 2002; Beehr et al., 2000; Henkens y Tazelaar, 1994; Henkens y Tazelaar, 1997; Koloski et al., 2001; Taylor y Shore, 1995). Hasta el momento, apenas se han realizado investigaciones sobre el punto hasta el cual las dolencias psicológicas juegan un papel en ella. Curiosa laguna cuando, desde luego en los Holanda, el número de personas que abandona el mercado de trabajo de forma prematura a consecuencia de una dolencia psicológica es mayor que el número de empleados que queda incapacitado para el trabajo por dolencias físicas (Houtman, De Jonge y Smulders, 2007).

El artículo se estructura de la siguiente manera: en la primera mitad, abordaremos el trasfondo teórico del burnout y los factores determinantes más importantes de este síndrome. En el apartado metodológico, presentaremos los datos y los instrumentos de medición utilizados. En el apartado siguiente, se expondrán los resulta- 
dos. Cerraremos con las conclusiones más importantes y con una reflexión sobre las implicaciones más significativas para la política organizativa.

\section{MARCO TEÓRICO}

En este apartado se aborda qué es el burnout, qué dimensiones hay que distinguir y cuáles son los factores determinantes más importantes. Asimismo, se abordan sucesivamente aspectos de la situación laboral, el apoyo social y, por último, la personalidad de un empleado.

\section{¿Qué es el burnout?}

El burnout es una disminución de la adaptación al estrés que va acompañada de disfunciones crónicas en el trabajo (Maslach, 1993) y se considera una consecuencia de la exposición prolongada al estrés laboral crónico (Shirom, 2003). El instrumento de medición del burnout más utilizado y validado es el Inventario del Burnout de Maslach (MBI) (Maslach y Jackson, 1981). El MBI incluye ítems que miden conjuntos de síntomas que forman parte del síndrome de burnout: agotamiento emocional, despersonalización y percepción de falta de realización personal (Shirom, 2003). Siguiendo el ejemplo de Maslach y Jackson, Schaufeli y Van Dierendonck (2000) desarrollaron una versión holandesa del MBI, la Escala del Burnout de Utrecht (UBOS), que incluye una versión general utilizable más allá de los grupos profesionales del sector servicios. De acuerdo con la definición de estos autores, el burnout tiene que ver con un síndrome que se caracteriza por tres dimensiones: 1) sentimientos de agotamiento emocional, 2) aumento de la despersonalización en el trabajo y 3) una percepción negativa de las propias competencias en el trabajo.

Los sentimientos de agotamiento emocional remiten a sentimientos de cansancio extremo y de agotamiento de las fuentes emocionales y físicas de una persona. La despersonalización remite a una actitud negativa e insensible o a una reacción excesivamente distante ante diferentes aspectos del trabajo, y representa el componente interpersonal del burnout (Shirom, 2003). El elemento de reducción de la propia eficacia representa el componente de autoevaluación del burnout. Remite a sentimientos de incompetencia y de falta de realización y productividad en el trabajo (Maslach y Jackson, 1981).

\section{Elementos determinantes del burnout}

\section{Características del trabajo}

Un modelo teórico que intenta explicar el surgimiento del burnout es la teoría de la "conservación de recursos" (COR) de Hobfoll y Freedy (1993). En opinión de Hobfoll y Freedy, las exigencias que se plantean en el trabajo (job demands) y los factores que pueden considerarse recursos (job resources) determinan la motivación para participar en el proceso de trabajo. La esencia de este modelo es que el entorno laboral y características del puesto de trabajo como la autonomía, los desafíos y las posibilidades de desarrollo (recursos), así como la carga laboral y los aspectos físicos del trabajo (exigencias), explican en buena medida el surgimiento del burnout (Halbesleben y Buckley, 2004).

Un segundo modelo teórico utilizado en la investigación del burnout es el modelo de Exigencias y Recursos Laborales 
(Demerouti, Bakker, Nachreiner y Schaufeli, 2001). En este modelo, se parte de la hipótesis de que el burnout se desarrolla en la medida en que las exigencias que se plantean en el trabajo son elevadas y la presencia de recursos es limitada. Las exigencias que se plantean en el trabajo remiten a aspectos físicos, sociales y organizativos que requieren un esfuerzo físico y mental constante y, por ello, están asociadas a costes psicológicos (por ejemplo, agotamiento emocional). Los recursos en el puesto de trabajo son aspectos que pueden resultar funcionales en la persecución de objetivos personales, que pueden contribuir a atenuar las exigencias laborales planteadas y que estimulan el crecimiento y el desarrollo personal (Demerouti et al., 2001). En la mayoría de los estudios sobre las exigencias en el puesto de trabajo en relación con el burnout, se ha constatado que lo que más influye en los sentimientos de agotamiento emocional y, en menor medida, en la despersonalización, es la carga laboral (Lee y Ashforth, 1996; Schaufeli y Enzmann, 1998). Entre los factores del puesto de trabajo que pueden considerarse recursos y que están asociados al burnout, cabe citar la falta de autonomía (Lee y Ashforth, 1996; Schaufeli y Bakker, 2007), la falta de posibilidades de desarrollo (Lee y Ashforth, 1996) y la ausencia de desafíos (Pines, 1993). En nuestra investigación, formulamos la hipótesis de que cuanto mayores son la dureza física del trabajo y la carga laboral y menores los desafíos, las posibilidades de desarrollo y la autonomía, mayor es la probabilidad de padecer burnout (hipótesis 1).

\section{Apoyo social}

Otra causa importante del surgimiento del burnout es la falta de apoyo social
(Schaufeli y Bakker, 2007). Las personas forman parte de diferentes redes y pueden ser miembros de diferentes grupos que se solapan entre sí, como la familia o el trabajo. Las redes sociales plantean exigencias a las personas, pero también pueden ofrecer apoyo (Pines y Aronson, 1988). El apoyo social juega un papel importante en relación con la salud de una persona y constituye una fuente significativa que cabe utilizar en la prevención del burnout (Baruch-Feldman, Brondolo, Ben-Dayan y Schwartz, 2002; Cordes y Dougherty, 1993; Halbesleben, 2006; Hendrix, Cantrell y Steel, 1988; Leiter, 1991; Sundin, Hochwälder, Bildt y Lisspers, 2007).

En relación con la explicación del burnout, se estima que el apoyo social, por parte, entre otros, de los directivos, los compañeros de trabajo y la pareja, es relevante (Demerouti et al., 2001; Halbesleben, 2006). Uno de los recursos que puede atenuar el estrés en el trabajo es el apoyo social de los directivos (Kickul y Posig, 2001; Schaufeli y Enzmann, 1998). La relación con los directivos puede servir de amortiguador frente al estrés en el trabajo, por ejemplo, dando feedback y ofreciendo trabajo desafiante (Schaufeli y Bakker, 2007). Por otro lado, este recurso puede influir directamente en las exigencias del trabajo, por ejemplo, cuando un directivo le pide a un empleado tener determinado rendimiento (Halbesleben, 2006). Los sentimientos de agotamiento emocional y distanciamiento pueden estar originados por problemas que los empleados experimentan con otras personas. Puede tratarse de problemas con los directivos, pero también con otros compañeros de trabajo. La falta de apoyo por parte de los compañeros de trabajo puede conllevar un aumento del riesgo de burnout (Schaufeli y Bakker, 2007). El burnout es un fenómeno relacio- 
nado con el trabajo que, sin embargo, no está exclusivamente circunscrito a la situación laboral (Cordes y Dougherty, 1993). Un buen matrimonio puede hacer que los empleados resistan mejor el estrés en el trabajo, porque sienten apoyo en el hogar. Por otro lado, el burnout también puede influir en la calidad del matrimonio (Halbesleben, 2006).

A partir de las teorías recogidas en la literatura con respecto a la relación entre el apoyo social y el burnout, formulamos la siguiente hipótesis: cuanto menor es el apoyo social de los compañeros de trabajo, los directivos y la pareja (cónyuge), más posibilidades hay de que los empleados padezcan burnout (hipótesis 2).

\section{Autoeficacia}

El tercer tipo de factores a partir de los cuales se origina el burnout tiene que ver con la personalidad del individuo. Se supone que hay diferencias individuales entre los empleados y su riesgo de burnout, aunque se enfrenten a situaciones idénticas en el trabajo y en la vida privada. Un rasgo de personalidad que se supone que está asociado al riesgo de burnout es la autoeficacia. La autoeficacia, de acuerdo con la definición de Bandura (1977) es: "la convicción de tener un control sobre las propias capacidades en acontecimientos que influyen en la propia vida". Bandura (1977) admite que las circunstancias del entorno del individuo no son siempre tan favorables y que, en tales casos, la autoeficacia no es suficiente para una adaptación positiva. No obstante, la manera en que las personas manejan esto es muy variable. Los individuos que tienen un nivel alto de autoeficacia se demostrarán más activos socialmente en la resolu- ción de problemas. Si estas personas no consiguen cambiar el entorno, es probable que busquen un entorno laboral en el que vayan a funcionar mejor. En cambio, las personas con un nivel bajo de autoeficacia tienden a reaccionar ante un entorno adverso adoptando una actitud apática, resignada y cínica. Por otro lado, los factores del entorno también influyen en la autoeficacia de una persona (Cherniss, 1993). Las personas con un fuerte sentimiento de autoeficacia experimentan menos estrés en situaciones amenazadoras o que suponen mucha presión que las personas con un sentimiento menos fuerte de autoeficacia. Para las personas con un fuerte sentimiento de autoeficacia, las situaciones resultan también menos estresantes si pueden sobrellevarlas bien. Prevemos, por lo tanto, que cuanto menores sean los sentimientos de autoeficacia experimentados por los empleados, mayor será su padecimiento de burnout (Shirom, 2003) (hipótesis 3).

\section{Burnout e intenciones de jubilación anticipada}

Una pregunta importante es hasta qué punto el distanciamiento mental del proceso de trabajo está relacionado con los proyectos de jubilación. ¿Está asociado el padecimiento de burnout a un fuerte deseo de dejar de trabajar lo más pronto posible o acaso esta relación es menos clara de lo que en ocasiones se supone? Hay diversos motivos por los cuales las personas que padecen burnout pueden tener intenciones más firmes de jubilación. En primer lugar, el burnout debilita el lazo con el trabajo. Se supone que las personas que tienen un lazo más débil con la situación laboral optan antes por la jubilación. La jubilación ofrece en estos casos a los empleados 
mayores la posibilidad de abandonar situaciones desagradables en el puesto de trabajo. Otro motivo por el cual el padecimiento de burnout puede llevar a una intención más firme de jubilación tiene que ver con la posible reducción de este síndrome una vez que se deja de trabajar. Es sabido que muchos empleados mayores optan por la jubilación porque se espera que dejar de trabajar sea bueno para la salud (Henkens, 1999) y que la jubilación pueda influir favorablemente en los hábitos que repercuten en la salud (Henkens et al., 2008). La jubilación sirve, por así decirlo, como inversión en la propia salud. Esto es particularmente así en el caso de los empleados que padecen burnout. Así pues, a partir de los citados razonamientos, prevemos que, cuando los empleados mayores padecen formas más agudas de burnout, las intenciones de jubilarse anticipadamente son también más firmes (hipótesis 4). Sabemos, sin embargo, que los proyectos de jubilación no sólo están influidos por la situación en el puesto de trabajo, sino también por las posibilidades financieras de la economía familiar y por la situación personal. Dentro de este capítulo, es sobre todo relevante el papel de la pareja.

\section{MÉTODO}

\section{Muestra y procedimiento}

Para este estudio se han utilizado datos recogidos en 2001 por el Nederlands Interdisciplinair Demografisch Instituut (NIDI) de La Haya (Henkens y Van Solinge, 2003). En la investigación, se trabajó con 2.927 empleados de 50 años o más y sus parejas. Los empleados trabajaban en cuatro compañías del sector privado y en la Administración pública. Los empleados recibieron en un sobre un cuestionario para ellos y otro para su pareja (en caso de haberla). El cuestionario incluía una carta del investigador con una explicación del objetivo de la investigación y una carta de recomendación de los directores de las compañías e institución pública participantes. En el cuestionario se presentaban diferentes aspectos relacionados con la jubilación. Junto a las características demográficas básicas del empleado, se recogía información sobre la situación laboral, la vivencia del trabajo, la salud y las intenciones de jubilación. En total, se han devuelto un 63 $\%$ de los cuestionarios enviados a los empleados, después de haber mandado un recordatorio dos veces. Las parejas (o cónyuges) han rellenado el cuestionario adjunto en casi todos los casos (94\%). En la investigación están representadas en suficiente medida diferentes categorías de empleados: tanto de baja como de alta cualificación, a tiempo completo y a tiempo parcial, hombres y mujeres. El grupo total estaba compuesto de 2.195 hombres (76 $\%)$ y 697 mujeres (24\%), de los cuales el $41 \%$ contaba con una cualificación baja, el $28 \%$, media y el $31 \%$, alta. La edad oscilaba en 2001 entre los 50 y los 65 años $(M=54,6 ; d t=2,81)$. Por último, se eliminaron 35 cuestionarios porque las personas encuestadas se habían equivocado en más de tres de los siete ítems del burnout dentro de las dimensiones de agotamiento emocional y despersonalización. Así pues, el grupo analizado final estaba compuesto por 2.892 personas encuestadas.

\section{Instrumentos y medidas}

\section{Burnout}

Las dos dimensiones del burnout analizadas se midieron con una versión abreviada de la Escala del Burnout de Utrecht 
(Schaufeli y Van Dierendonck, 2000), de la que se eligieron los siguientes ítems:

Agotamiento emocional. El agotamiento emocional constaba de cuatro ítems: "trabajar todo el día constituye una carga pesada para mí", "me siento consumido por el trabajo", "al final del día me siento vacío" y "me siento cansado cuando me levanto por las mañanas y vuelvo a tener un día de trabajo por delante". $\alpha=0,85$.

Despersonalización. La despersonalización constaba de tres ítems: "dudo del sentido de mi trabajo", "noto que me he distanciado demasiado de mi trabajo" y "mi trabajo ya no me entusiasma tanto como antes". $\alpha=0,71$.

Las personas encuestadas podían dar sus contestaciones con respecto a estas dos dimensiones dentro de una escala de respuestas de 7 puntos, que iba de $0=$ "nunca", 1 = "esporádicamente", 2 = "de vez en cuando", 3 = "con regularidad", 4 = "con frecuencia", 5 = "con mucha frecuencia", hasta $6=$ "siempre". Para las dimensiones del burnout se utilizaron puntuaciones no estandarizadas.

\section{Intencionalidad}

Intenciones de jubilación anticipada. Las intenciones de jubilación anticipada se midieron a partir de cuatro preguntas: 1) "¿tiene planes de dejar de trabajar antes de cumplir los 65 años?; 2) "¿a qué edad quiere dejar de trabajar?”; 3) “¿quiere seguir trabajando después de los 61 años?" y 4) "si dependiera de usted, ¿a qué edad preferiría dejar de trabajar?". Los tipos de respuesta variaban por ítem: en el primer ítem, los encuestados podían elegir entre las respuestas "sî" y "no, aún no". En el segundo ítem, los encuestados podían rellenar la edad; el tercer ítem tenía seis tipos de respuesta, que iban desde "no, desde luego que no" a "sí, desde luego que sí" y "ya lo hago", y el cuarto ítem se podía rellenar con la edad en la que uno preferiría dejar de trabajar. Como los diferentes ítems no tenían las mismas posibilidades de respuesta, se procedió a estandarizar y, a continuación, a enumerar los ítems en la escala. Esta escala estandarizada se transformó luego linealmente en una escala con un intervalo de resultados que iba del 1 al 10. El número uno significaba "intencionalidad mínima de jubilación” y el número diez "intenciones muy claras de jubilación". $\alpha=0,84$. La puntuación media de las intenciones de jubilación era $M=7,32$ y la desviación típica era $d t=1,80$.

\section{Características del trabajo}

Carga de trabajo. La carga de trabajo se midió con cuatro ítems: 1) “¿con cuánta frecuencia tiene problemas en su trabajo con las siguientes cuestiones: carga de trabajo?". Los encuestados podían dar una respuesta dentro de una escala que iba de "con mucha frecuencia" (1) a "nunca" (4). El segundo ítem constaba de la frase: "la cantidad de trabajo a veces es demasiado grande para poder hacer todo bien"; el tercer ítem: "con frecuencia tengo que esmerarme para poder hacer todo"; y el cuarto ítem: "a veces la carga de trabajo es tal que genera tensión”. La escala de respuestas de cinco puntos para estas frases variaba de "totalmente de acuerdo" (1) a "en total desacuerdo" (5).

Para esta escala, se invirtieron, estandarizaron y sumaron los resultados de todos 
los ítems. Una puntuación alta en la carga de trabajo significaba una carga de trabajo elevada. $\alpha=0,80$.

Dureza física del trabajo: se midió con un ítem. La frase de este ítem era "mi trabajo es duro físicamente". La respuesta se podía marcar en una escala de respuestas de cinco puntos que iba de "totalmente de acuerdo" (1) a "en total desacuerdo" (5). Los resultados de este ítem se invirtieron, de manera que una puntuación alta significaba un trabajo duro físicamente.

Desafíos. La escala de desafíos constaba de tres ítems, que se marcaban en una escala de respuestas de cinco puntos de "totalmente de acuerdo" (1) a "en total desacuerdo" (5). El primer ítem constaba de la frase: "en los últimos años, el trabajo que hago no me ofrece apenas desafíos"; el segundo ítem: "mi trabajo incluye muchas tareas desafiantes"; y el tercer ítem: "en los últimos años, el trabajo que hago se ha convertido en rutina". Para esta escala, se invirtió el resultado del segundo ítem, de manera que una puntuación alta significaba que los empleados tenían muchos desafíos en su trabajo. Los ítems para esta escala primero se estandarizaron al nivel de cada ítem y, a continuación, se sumaron. $\alpha=0,76$.

Posibilidades de desarrollo. Se midieron a través de dos ítems: 1) "mi trabajo ofrece buenas oportunidades de promoción" y 2) "mi trabajo no ofrece muchas más posibilidades de desarrollo", con una inversión de los resultados del primer ítem. Los tipos de respuesta para estas frases iban de "totalmente de acuerdo" (1) a "en total desacuerdo" (5). Una puntuación alta significaba muchas posibilidades de desarrollo. Ambos ítems se estandarizaron primero y luego se sumaron. $\alpha=0,61$.

Autonomía. La autonomía se midió a través de un ítem: "dentro de unos límites, puedo organizar mi trabajo por mi cuenta". El tipo de respuesta para este ítem era una escala de respuestas de cinco puntos que iba de (1) "totalmente de acuerdo" a (5) "en total desacuerdo". El resultado de este ítem se invirtió. Una puntuación alta significaba mucha autonomía.

\section{Apoyo social}

Relación con los compañeros de trabajo. Se midió con dos ítems y se procedió a estandarizar y sumar los resultados al nivel de cada ítem antes de integrarlos en la escala: 1) “ ¿con cuánta frecuencia tiene problemas en su trabajo con las siguientes cuestiones: trato con los compañeros de trabajo?" y 2) "la relación con mis compañeros de trabajo es excelente". Las posibilidades de respuesta para el primer ítem variaban de "con mucha frecuencia" (1) a "nunca" (4). La respuesta para el segundo ítem se marcaba en una escala de cinco puntos que iba de "totalmente de acuerdo" (1) a "en total desacuerdo" (5). Para esta escala, se recodificaron los resultados del segundo ítem, de manera que, al final, una puntuación alta en apoyo social significaba mucho apoyo. $\alpha=0,59$.

Apoyo social de los directivos. Se midió con un ítem: "mi jefe/directivo me estimula para que mantenga al día los conocimientos que requiere mi trabajo". Las respuestas se marcaban en una escala de cinco puntos que iba de "totalmente de acuerdo" (1) a "en total desacuerdo" (5). La puntuación para este ítem se invirtió: 
una puntuación alta significaba mucho apoyo social.

Relaciones conyugales. Las relaciones conyugales se midieron en función de seis variables ficticias que indicaban cuántas actividades conjuntas realizaban los empleados y sus parejas. Esta cantidad puede considerarse un indicador de la calidad del matrimonio (Davey y Szinovacz, 2004). La pregunta se le planteó a la pareja de los empleados y decía así: “¿En cuáles de las siguientes actividades pasan ustedes tiempo libre juntos?" y constaba de 15 actividades en total: a) aficiones, b) la familia, c) los nietos (si los tiene), d) viajes/vacaciones, e) trabajo voluntario, f) leer, g) estudio/cursos, h) ver la televisión, i) ir de compras, j) pasear/montar en bici/hacer deporte, $\mathrm{k}$ ) visitas familiares, 1) arreglar la casa/cuidar el jardín, m) apoyo o ayuda de los padres, los hijos u otros familiares, $n$ ) salir, ir al teatro, al cine. Las posibilidades de respuesta eran "sí" o "no". De los resultados se desprendía que el $8 \%$ de los encuestados había marcado con una cruz menos de seis actividades, el $29 \%$, entre 7 y 9 , el $31 \%$ del grupo total realizaba entre 10 y 11 actividades en común y el $14 \%$, entre 12 y 15 .

\section{Personalidad}

Autoeficacia. Esta escala es una versión abreviada de la Escala General de Autoeficacia (Scherer et al., 1982) y se midió con cinco ítems. Los ítems se estandarizaron al nivel de cada ítem y, a continuación, se sumaron: 1) "dudo de mí mismo", 2) "cuando quiero algo de verdad, me suele salir mal", 3) "cuando tengo la impresión de que algo es complicado, no lo empiezo", 4) "tengo dificultades para resolver bien los problemas que se me pre- sentan en la vida" y 5) "cuando hago planes, estoy convencido de que lograré llevarlos a cabo". Las respuestas se marcaban en una escala de cinco puntos que iba de "totalmente de acuerdo" (1) a "en total desacuerdo" (5). El resultado del quinto ítem se invirtió. Una puntuación alta significaba un nivel alto de autoeficacia. $\alpha=0,65$.

\section{Análisis de datos}

Para responder a la pregunta de qué factores estaban asociados al agotamiento emocional y la despersonalización se llevaron a cabo análisis de regresión, con las dos dimensiones medidas del burnout como variable dependiente y las características del trabajo, el apoyo social y la autoeficacia como variables independientes. También se utilizó el análisis de regresión en el estudio de las conexiones entre las dos dimensiones del burnout y las intenciones de jubilación anticipada. Las variables de control de este estudio eran: edad (medida como variable continua), sexo (medido como variable ficticia: varón $=0$, mujer = 1) y formación, que se midió como variable continua, con una escala de respuestas que iba de 1 (educación primaria) a 7 (universidad o educación superior).

\section{RESULTADOS}

En la Tabla 1, reflejamos los porcentajes de las puntuaciones relativas a las dimensiones de agotamiento emocional y despersonalización propias del burnout. Estas puntuaciones se comparan con las pautas de la Escala del Burnout de Utrecht (UBOS) para trabajadores en activo, es decir, en principio, «empleados sanos». Para la estandarización de la UBOS se ha 
Burnout e intenciones de jubilación anticipada entre empleados mayores

optado por una división en cinco bloques: $0,44<\mathrm{r}<0,51)$ (Schaufeli y Van Dieren«muy bajo», «bajo», «medio», «alto»y donck, 2000).

Tabla 1. Porcentaje de las puntuaciones para las dos dimensiones del burnout en empleados de 50 años y mayores

\begin{tabular}{|lcc|}
\hline & $\begin{array}{c}\text { Agotamiento } \\
\text { emocional }\end{array}$ & Despersonalización \\
\hline Puntuación & & $17 \%$ \\
Muy bajo & $7 \%$ & $13 \%$ \\
Bajo & $23 \%$ & $48 \%$ \\
Medio & $47 \%$ & $19 \%$ \\
Alto & $19 \%$ & $3 \%$ \\
Muy alto & $4 \%$ & 1,15 \\
M & 1,54 & 1,00 \\
dt & 1,11 & $100 \%$ \\
& $100 \%$ & $(\mathrm{~N}=2.892)$ \\
\hline
\end{tabular}

Fuente: investigación del NIDI (2001): Trabajo, envejecimiento y jubilación.

«muy alto». En las escalas individuales, una puntuación (muy) alta en agotamiento emocional y una puntuación (muy) alta en despersonalización es indicadora de burnout. La media del grupo estándar es, para el agotamiento emocional, de 1,78 y, para la despersonalización, de 1,34 (Schaufeli y Van Dierendonck, 2000). En nuestro grupo de investigación las medias son menores: de 1,54 y 1,15 respectivamente. De la tabla se desprende que, en comparación con el grupo estándar, un $23 \%$ de los colaboradores tenían una puntuación (muy) alta en agotamiento emocional y un $22 \%$ tenían una puntuación (muy) alta en despersonalización. La correlación entre agotamiento emocional y despersonalización $(r=0,52)$ entra dentro del rango obtenido en otros estudios ( $\mathrm{r}$ media $=0,48$;
En la Tabla 2, pueden verse los resultados del análisis de regresión que explican las dimensiones de agotamiento emocional y despersonalización del burnout. En la primera columna, aparecen representados los resultados de los efectos que tienen las características del trabajo, el apoyo social y la autoeficacia sobre la dimensión del agotamiento emocional. De los coeficientes de regresión estandarizados se desprende que, de las características del trabajo, la que tiene un efecto más fuerte sobre los sentimientos de agotamiento emocional es la carga de trabajo $(\beta=0,48, \mathrm{p}<0,001)$ : cuanto mayor es la carga de trabajo, más desgastados se sienten los empleados. La dureza física del trabajo juega un papel mucho menor $(\beta=0,10, \mathrm{p}<0,001)$. Entre los aspectos gratificantes del trabajo, los 
Tabla 2. Análisis de regresión para explicar las dimensiones de agotamiento emocional y despersonalización del burnout en función de las características del trabajo, el apoyo social y la autoeficacia

\begin{tabular}{|c|c|c|c|c|}
\hline \multirow{3}{*}{\begin{tabular}{|l} 
\\
Variables de control
\end{tabular}} & \multicolumn{2}{|c|}{$\begin{array}{l}\text { Modelo } 1 \\
\text { Agotamiento emocional }\end{array}$} & \multicolumn{2}{|c|}{$\begin{array}{l}\text { Modelo } 2 \\
\text { Despersonalización }\end{array}$} \\
\hline & \multirow[t]{2}{*}{$\beta$} & \multirow[t]{2}{*}{$\mathrm{t}$} & \multirow[t]{2}{*}{$\beta$} & \multirow[t]{2}{*}{$\mathrm{t}$} \\
\hline & & & & \\
\hline edad & $-0,02$ & $-1,64$ & 0,03 & 1,69 \\
\hline $\operatorname{sexo}$ & $-0,04 * *$ & $-2,77$ & $0,12^{* * * *}$ & 7,46 \\
\hline formación & $0,05^{* *}$ & 2,90 & $0,15^{* * * *}$ & 8,86 \\
\hline \multicolumn{5}{|l|}{ Características del trabajo } \\
\hline carga de trabajo & $0,48^{* * *}$ & 29,23 & 0,20 *** & 12,01 \\
\hline dureza física del trabajo & $0,10^{* * *}$ & 6,01 & $-0,07 * * *$ & $-4,28$ \\
\hline desafíos & $-0,15^{* * *}$ & $-8,57$ & $-0,40 * * *$ & $-21,60$ \\
\hline posibilidades de desarrollo & $-0,06 * * *$ & $-3,64$ & $-0,02$ & $-1,30$ \\
\hline autonomía & 0,00 & 0,19 & 0,00 & 0,05 \\
\hline \multicolumn{5}{|l|}{ Apoyo social } \\
\hline de los compañeros de trabajo & $-0,08 * * *$ & $-5,44$ & $-0,12 * * *$ & $-7,82$ \\
\hline de los directivos & $-0,03$ & $-1,94$ & $-0,08^{* * *} *$ & $-4,83$ \\
\hline \multicolumn{5}{|l|}{ Relaciones conyugales } \\
\hline \multicolumn{5}{|l|}{ sin pareja (ref. cat.) } \\
\hline pocas actividades en común & $-0,03$ & $-1,67$ & $-0,03$ & $-1,37$ \\
\hline \multicolumn{4}{|l|}{ común } & 0,49 \\
\hline bastantes actividades en común & $-0,04$ & $-1,90$ & 0,00 & $-0,15$ \\
\hline muchas actividades en común & $-0,05$ & $-2,28$ & $-0,01$ & $-0,32$ \\
\hline sin cuestionario de la pareja & $-0,02$ & $-1,11$ & $-0,02$ & $-0,87$ \\
\hline \multicolumn{5}{|l|}{ Rasgos de la personalidad } \\
\hline autoeficacia & $-0,06 * * *$ & $-3,69$ & $-0,13^{* * * *}$ & $-7,98$ \\
\hline $\mathrm{N}=$ & 2.892 & & 2.892 & \\
\hline $\mathrm{R}^{2}$ & $40 \%$ & & $36 \%$ & \\
\hline
\end{tabular}

leyenda: $* \mathrm{p}<0,05 ; * * \mathrm{p}<0,01 ; * * * \mathrm{p}<0,001$. 
desafíos son lo más relevante $(\beta=-0,15, \mathrm{p}$ $<0,001)$. Los empleados experimentaban un agotamiento emocional mayor cuanto menos desafiante era su trabajo. Aunque el efecto de las posibilidades percibidas de desarrollo es reducido $(\beta=-0,06, \mathrm{p}<$ $0,001)$, unas posibilidades de desarrollo menores llevaban a mayores sentimientos de agotamiento emocional. Por lo que se refiere al apoyo social, se desprende que sólo el apoyo de los compañeros de trabajo tiene un efecto, aunque reducido, en el agotamiento emocional $(\beta=-0,08, \mathrm{p}<$ $0,001)$ : cuanto más clara es la presencia de apoyo, menor es el burnout que padecen los empleados. También la autoeficacia tiene un efecto significativo pero reducido $(\beta=-0,06, p<0,001)$. El coeficiente total de determinación para el modelo es de un $40 \%$.

El análisis de regresión realizado para explicar la despersonalización aparece recogido en la segunda columna. Por lo que se refiere al efecto de las características del trabajo sobre la dimensión de despersonalización, se desprende que el efecto que más peso tiene es aquél correspondiente a los desafíos que el empleado encuentra en el puesto de trabajo ( $\beta=$ $0,40, \mathrm{p}<0,001)$. Un empleo poco desafiante aumenta las probabilidades de burnout. También la carga de trabajo influye en las probabilidades de que los empleados mayores se distancien más con respecto a su trabajo $(\beta=0,20, \mathrm{p}<0,001)$. Los empleados que se enfrentan a una gran carga de trabajo toman más distancia de su trabajo que los empleados que se enfrentan a una carga de trabajo menor. Los efectos de la autonomía y de las posibilidades de desarrollo no son significativos. Por lo que se refiere al apoyo social, resulta ante todo relevante el papel de los compañeros de trabajo $(\beta=-0,12, \mathrm{p}<0,001)$ : los empleados que disfrutan de más apoyo de sus compañeros de trabajo toman menos distancia de su trabajo que los empleados que cuentan en menor medida con este apoyo. El efecto del apoyo social de los directivos y de la pareja no es significativo. Por último, estudiamos los efectos de la autoeficacia sobre la despersonalización. Los empleados que sienten menos autoeficacia se distancian más de su trabajo que los empleados que sienten más autoeficacia $(\beta=-0,13, \mathrm{p}<0,001)$. Además, se descubrió que tenían un efecto significativo las variables de control de sexo $(\beta=0,12, p<0,001)$ y formación $(\beta=0,15, \mathrm{p}<0,001)$. El coeficiente de determinación para la despersonalización es de un $36 \%$.

Los resultados permiten ver que el agotamiento emocional y la despersonalización son reacciones ante diferentes aspectos del trabajo, el entorno (social) y la personalidad individual: el agotamiento emocional se explica en su mayor parte por una gran carga de trabajo y, en menor medida, por la falta de desafíos y por la dureza física del trabajo. La despersonalización se explica principalmente por la falta de desafíos y por una gran carga de trabajo y, en menor medida, por un déficit de apoyo social por parte de los compañeros de trabajo y por una autoeficacia menor. No descubrimos ningún efecto significativo del número de actividades realizadas en común con la pareja (o cónyuge).

Los resultados para explicar las intenciones de jubilación aparecen reflejados en la Tabla 3. De la tabla se desprende que los empleados que se sienten más desgastados $(\beta=0,22, p<0,001)$ y que más se distancian del trabajo $(\beta=0,12$, $p$ $<0,001)$ tienen un deseo más fuerte de jubilación que los empleados que no sien- 
Tabla 3. Análisis de regresión para explicar las intenciones de jubilación anticipada en relación con las dos dimensiones del burnout y tres factores determinantes (características del trabajo, apoyo social y autoeficacia)

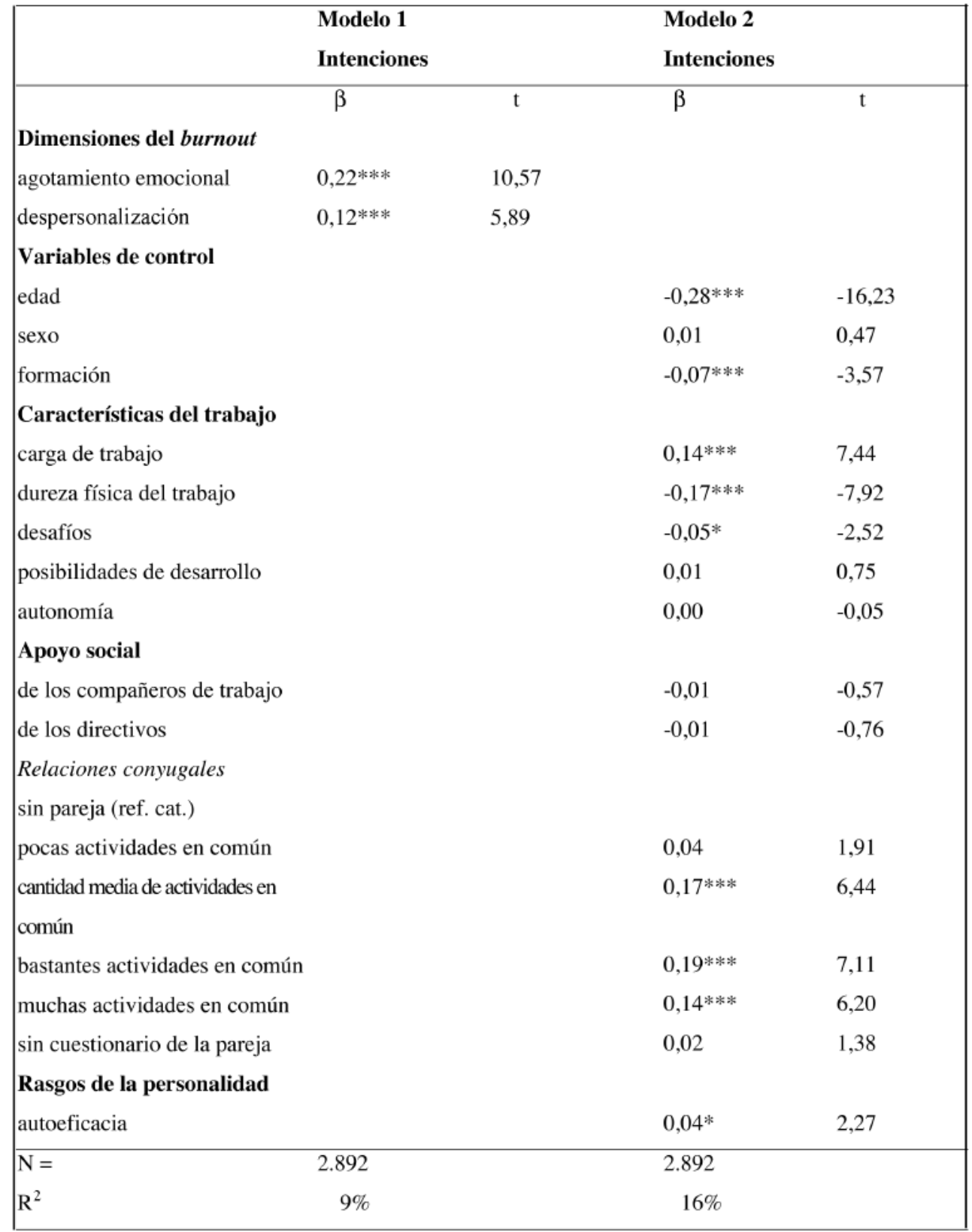

leyenda: $* \mathrm{p}<0,05 ; * * \mathrm{p}<0,01 ; * * * \mathrm{p}<0,001$. 
ten estos malestares o los sienten en menor medida. El coeficiente de determinación para las dos dimensiones analizadas del burnout en relación con las intenciones de jubilación es de un $9 \%$. En la segunda columna se analiza la relación de las características del trabajo, el apoyo social y la autoeficacia con las intenciones de los empleados mayores de jubilarse de manera anticipada. De las variables dentro del bloque de características del trabajo, se desprende que la carga de trabajo y los desafíos contribuyen de manera significativa a la explicación de las intenciones de jubilación $(\beta=0,14, \mathrm{p}<0,001$ en $\beta=-0,17, p<0,001)$. Los empleados que se enfrentan a una carga de trabajo mayor y que encuentran el trabajo menos desafiante tienen una intención más firme de dejar el proceso de trabajo antes de tiempo que los empleados que tienen una carga de trabajo menor y hallan más desafíos. En este caso, no se detecta ningún efecto significativo de las relaciones sociales con los compañeros de trabajo ni del apoyo social de los directivos. El apoyo obtenido del matrimonio sí que tiene importancia en la explicación de las intenciones de jubilación. Los empleados que realizan más actividades con su pareja tienen una intención más firme de jubilación que los empleados que no tienen pareja. En cambio, los empleados que apenas comparten actividades con su pareja no parecen tener una intención más firme de jubilación que los empleados que no tienen pareja. El efecto de la autoeficacia es significativo pero reducido $(\beta=$ $0,04, \mathrm{p}<0,001)$. De las variables de control, se desprende que el efecto de la edad ( $\beta=-0,28, p<0,001)$ es relevante. Junto con las variables de control, las características de trabajo, el apoyo social y la autoeficacia explican un $16 \%$ de las intenciones de jubilación.
Los resultados de la influencia conjunta del agotamiento emocional y la despersonalización, por un lado, y de las características del trabajo, el apoyo social y la autoeficacia, por otro, aparecen representados en la tercera columna. Del análisis se desprende que los porcentajes de agotamiento emocional y despersonalización median la relación entre los tres factores explicativos y las intenciones de jubilación. El papel mediador del burnout parece actuar en particular en el caso de dos características del trabajo: la carga de trabajo y los desafíos. La fuerza del efecto directo de estas dos características del trabajo sobre las intenciones de jubilación disminuye en la medida en que incluimos el agotamiento emocional y la despersonalización en la explicación.

\section{DISCUSIÓN}

En este artículo se da cuenta de una investigación realizada con 2.892 empleados mayores de Holanda sobre el padecimiento de burnout y la influencia que éste tiene en las intenciones de retirarse del proceso de trabajo. En la bibliografía internacional, se reconoce que la jubilación supone cada vez menos un cambio brusco de una vida de trabajo a una vida de ocio. Por un lado, es más frecuente que las personas se mantengan activas pese a todo en el mercado de trabajo. Por otro lado, hay personas que se despiden mentalmente del trabajo mucho antes de haber dejado de trabajar de manera definitiva. Una forma especial de distanciamiento mental del proceso de trabajo es el burnout. En este estudio, se investiga en primer lugar qué conexiones existen entre las características del trabajo, el apoyo social y la autoeficacia, por una parte, y dos dimensiones del burnout (agotamiento 
emocional y despersonalización), por otra. En segundo lugar, se investiga qué conexión existe entre estas dimensiones, los tres grupos de factores explicativos y las intenciones de los empleados mayores de jubilarse de manera anticipada. Una conclusión importante es que, en la explicación del agotamiento emocional, predomina la carga de trabajo elevada y, en menor medida, la falta de desafíos y la dureza física del trabajo. La despersonalización se explica ante todo por la falta de desafíos en el trabajo y, en menor medida, por la carga de trabajo elevada, la falta de apoyo social de los compañeros de trabajo y una autoeficacia más reducida. Estos resultados coinciden en gran medida con las conclusiones de la investigación de Lee y Ashforth (1996). En un meta-análisis realizado ante todo con empleados del sector servicios, estos investigadores descubrieron que el agotamiento emocional está fundamentalmente relacionado con la carga de trabajo. A su vez, la despersonalización está relacionada, aunque en menor medida, con fuentes en el trabajo, como el apoyo social. La hipótesis de Demerouti et al. (2001) es que el síndrome de burnout se desarrolla tanto si las exigencias del trabajo son altas como si la cantidad de fuentes en el trabajo es limitada. De nuestra investigación se desprende que juegan ante todo un papel importante en este proceso la carga de trabajo y los desafíos. Otros factores, como la dureza física del trabajo y, entre las diferentes fuentes, el apoyo social, parecen contribuir de manera discreta a la explicación del burnout. A continuación se analiza cómo se relacionan el agotamiento emocional y la despersonalización con las intenciones de los empleados mayores de jubilarse de manera anticipada. Para ello, se parte de la hipótesis de que cuanto más agotamiento emocional experimentan los empleados y más se distancian del trabajo, más firmes son sus intenciones de jubilación. De acuerdo con nuestras expectativas, constatamos que los empleados que experimentaban más agotamiento emocional y que más se distanciaban del trabajo, tenían una intención más firme de jubilarse que los empleados que no padecían este tipo de malestares. Sin embargo, a la luz del coeficiente de determinación, no se puede decir que la relación entre las dimensiones de agotamiento emocional y despersonalización y las intenciones de jubilación sea fuerte.

Del análisis se desprende ante todo que, junto a los efectos del agotamiento emocional y de la despersonalización sobre las intenciones de jubilación, influyen particularmente en estas intenciones las relaciones conyugales. Los empleados que realizaban más actividades en común con su pareja tenían intenciones de jubilación más firmes que los empleados que apenas compartían actividades. Este segundo grupo de empleados no se distinguía en sus intenciones de jubilación del grupo de empleados sin pareja. Este resultado llama especialmente la atención puesto que no se encontraron relaciones significativas entre la calidad del matrimonio y las dos dimensiones medidas del burnout.

Parece, pues, que el burnout y las intenciones de jubilación anticipada son en buena medida dos procesos diferentes. El proceso de agotamiento emocional y distanciamiento del trabajo propio del burnout parece estar determinado ante todo por características del trabajo como la carga de trabajo elevada y la ausencia excesiva de desafíos y, en cierta menor medida, por la falta de apoyo social de los compañeros de trabajo y la autoeficacia. Por el contrario, las intenciones de jubila- 
ción parecen explicarse en buena medida en función de factores externos al trabajo. La relación con la pareja parece determinar en una proporción importante la actitud del empleado con respecto a la jubilación, pero parece bastante evidente que no guarda ninguna relación con el burnout.

Esta investigación tiene una serie de limitaciones. En primer lugar, en esta investigación no se maneja un análisis transectorial. La segunda limitación es el sesgo de la selección: como los empleados más sanos se mantienen en el proceso de trabajo, los resultados pueden conllevar una subestimación de los efectos del burnout sobre las intenciones de jubilación. Hay una tercera limitación en la posibilidad de generalizar los resultados de la investigación a otros grupos de empleados mayores. La presente investigación se realizó dentro de cuatro grandes organizaciones privadas en el sector público y de una gran institución de la Administración Pública. Queda aún por investigar en qué medida se presenta el burnout entre los empleados mayores de las pequeñas organizaciones, así como las intenciones de jubilación anticipada de estos empleados. La cuarta limitación es que, para diversas variables independientes, se ha utilizado un ítem. Con la utilización de un único ítem, la fiabilidad es incierta.

En los últimos años, la administración ha adoptado medidas para promover la participación laboral de los empleados mayores, entre otras cosas, haciendo poco atractivas las normas de la jubilación anticipada, la pensión de prejubilación y la pensión puente. Hasta hace poco, los empresarios se mantenían considerablemente indiferentes con respecto a conservar durante más tiempo los esfuerzos de las personas mayores. Las decisiones de jubilación se consideraban un asunto privado y se suponía que, en principio, los empleados hacían uso de las posibilidades de jubilación. Para los empleados mentalmente desenganchados, las puertas de la jubilación anticipada estaban abiertas de par en par y, ya se tratase de una salida voluntaria u obligada, se trataba de una práctica habitual. Con el recorte presupuestario de las normas de jubilación, parece haber llegado el fin de estas situaciones. Este artículo permite ver que los empresarios ya no pueden presuponer de manera automática que aquellos empleados de los que preverían (o incluso esperarían) una retirada anticipada del mercado de trabajo decidan en efecto llevarla a cabo. Hasta ahora, los directivos se situaban fundamentalmente en la misma línea de banda con respecto al final de la carrera profesional de sus empleados. Sin embargo, en el futuro, deberían dedicar más esfuerzos a conseguir una dedicación satisfactoria de aquellos de sus empleados que se están haciendo mayores. Queda por ver hasta qué punto la actual política de cuidado hacia los empleados mayores contiene puntos de referencia para el deseado cambio cultural. Las normas para mayores y todos los tipos de extras en forma de días libres y obligaciones reducidas pueden contar con mucha simpatía entre los empleados. Sin embargo, los empresarios de Holanda sufren los inconvenientes de esta política de cuidado (véase Van Dalen et al., 2007). Estos inconvenientes no sólo residen en los costes financieros y en la disponibilidad reducida de los mayores. Los empresarios constatan también que más libertad no significa automáticamente menos estrés ni, en absoluto, que el trabajo se haga más atractivo para los empleados. Para conseguir esto último, parece indispensable encontrar nuevos caminos para dar un impulso al trabajo al final de 
la carrera profesional. La formación no es más que un aspecto de esta activación. Más importancia parece tener el aumento de la movilidad laboral de los mayores, sin que vaya acompañada de una pérdida demasiado grande de seguridad en el trabajo o en los ingresos. "Con una nueva función o puesto de trabajo, llega un nuevo desafío» es un adagio que parece también válido al final de la carrera profesional. Esto exige esfuerzos no sólo por parte del empresario, que debe buscar alternativas al despido de los empleados mayores. Exige también esfuerzos por parte de los propios empleados mayores, que deberán desprenderse de una parte de sus seguridades adquiridas para poder encontrar nuevos desafíos.

\section{BIBLIOGRAFÍA}

Adams, G.A. (1999). Career-related variables and planned retirement age: An extension of Beehr's model. Journal of Vocational Behavior, 55, 221-235.

Adams, G.A., Prescher J., Beehr, T.A. y Lepisto, L. (2002). Applying work-role attachment theory to retirement decisionmaking. International Journal of Aging and Human Development, 54, 125-137.

Bakker Comission Report (2008). Be Aware. Employment, Pensions, and Benefits.

Bandura, A. (1977). Self-efficacy: Toward a unifying theory of behavioral change. Psychological Review, 84, 191215.

Baruch-Feldman, C., Brondolo, E., BenDayan, D. y Schwartz, J. (2002). Sources of social support and burnout, job satisfac- tion, and productivity. Journal of Occupational Health Psychology, 7, 84-93.

Beehr, T.A., Glazer, S., Nielson, N.L. y Farmer, S.J. (2000). Work and nonwork predictors of employees' retirement ages. Journal of Vocational Behavior, 57, 206225.

Cherniss, C. (1993). Role of professional self-efficacy in the etiology and amelioration of burnout. En W.B. Schaufeli, C. Maslach y T. Marek (Eds.), Professional burnout: Recent developments in theory and research (pp. 135150). Washington, DC: Taylor \& Francis.

Cordes, C.L. y Dougherty, T.W. (1993). A review and an integration of research on job burnout. Academy of Management Review, 18, 621-656.

Dalen, H.P van, Henkens, K. en Schippers, J.J. (2007). Oudere werknemers door de lens van de werkgever [Empleados mayores desde la óptica del empresario], NIDI-rapport 74.

Davey, A. y Szinovacz, M.E. (2004). Dimensions of marital quality and retirement. Journal of Family Issues, 25, 431464.

Demerouti, E., Bakker, A.B., Nachreiner, F. y Schaufeli, W.B. (2001). The jobdemands-resources model of burnout. Journal of Applied Psychology, 86, 499512.

Halbesleben, J.R.B. (2006). Sources of social support and burnout: A meta-analytic test of the conservation of resources model. Journal of Applied Psychology, 91, 1134-1145. 
Burnout e intenciones de jubilación anticipada entre empleados mayores

Halbesleben, J.R.B. y Buckley, M.R. (2004). Burnout in organizational life. Journal of Management, 30, 859-879.

Hanisch, K.A. y Hulin, C.L. (1990). Job attitudes and organizational withdrawal: An examination of retirement and other voluntary withdrawal behaviors. Journal of Vocational Behavior, 37, 60-78.

Hendrix, W.H., Cantrell, R.S. y Steel, R.P. (1988). Effects of social support on the stress-burnout relationship. Journal of Business and Psychology, 3(1), 67-73.

Henkens, K., (1999). Retirement intentions and spousal support: A multi-actor approach. Journal of Gerontology: Social Sciences, 54B, S63-S73.

Henkens, K. y Solinge, H. van (2002), Spousal influences on the decision to retire, International Journal of Sociology, 32(2), 55-74.

Henkens, K. y Solinge, H. van (2003). Het eindspel: werknemers, hun partners en leidinggevenden over uittreden uit het arbeidsproces. Assen: Koninklijke van Gorkum B.V.

Henkens, K., Solinge, H. van y Gallo,W. (2008), Effects of retirement voluntariness on health behaviors: changes in smoking, drinking and physical activity among Dutch older workers, European Journal of Public Health, 18(6):644-649.

Henkens, K. y Tazelaar, F. (1994). Early retirement of civil servants in the Netherlands. Journal of Applied Social Psychology, 24, 1927-1943.

Henkens, K. y Tazelaar, F. (1997).
Explaining retirement decisions of civil servants in the Netherlands: Intentions, behavior, and the discrepancy between the two. Research on Aging, 19, 139-173.

Hobfoll, S.E. y Freedy, J. (1993). Conservation of resources: A general stress theory applied to burnout. En W.B. Schaufeli, C. Maslach y T. Marek (Eds.), Professional burnout: Recent developments in theory and research (pp. 115129). Washington, DC: Taylor \& Francis.

Houtman I., Jonge de, R. y Smulders, P. (2007). De epidemiologie van werkgerelateerde psychische aandoeningen en klachten [Epidemiología de las afecciones y dolencias psíquicas relacionadas con el trabajo]. En W.B. Schaufeli y A. Bakker (Eds.), De psychologie van arbeid en gezondheid [Psicología del trabajo y la salud] (pp. 267285). Houten: Bohn Stafleu Van Loghum.

Kickul, J. y Posig M. (2001). Supervisory emotional support and burnout: An explanation of reverse buffering effects. Journal of Managerial Issues, 13, 328344.

Koloski, K., Ekerdt, D. y DeViney, S. (2001). The role of job-related rewards in retirement planning. Journal of Gerontology: Psychological Sciences, 56B, 160-169.

Lee, R.T. y Ashforth, B.E. (1996). A meta-analytic examination of the correlates of the three dimensions of job burnout. Journal of Applied Psychology, 81, 123-133.

Leiter, M.P. (1991). Coping patterns as predictors of burnout: The function of control and escapist coping patterns. Journal of Occupational Behavior, 12, 123-144. 
Leiter, M.P. (1993). Burnout as a developmental process: Consideration of models. En W.B. Schaufeli, C. Maslach y T. Marek (Eds.), Professional burnout: Recent developments in theory and research (pp. 237-250). Washington, DC: Taylor \& Francis.

Maslach, C. (1993). Burnout: A multidimensional perspective. En W.B. Schaufeli, C. Maslach y T. Marek (Eds.), Professional burnout: Recent developments in theory and research (pp. 19-32). Washington, DC: Taylor \& Francis.

Maslach, C. (2000). Burnout: oorzaken, gevolgen en remedies (The truth about burnout) [Burnout. Causas, consecuencias y remedios (La verdad sobre el burnout)]. Amsterdam: Contact.

Maslach, C. (2003). Job burnout: New directions in research and intervention. American Psychological Science, 12, 189192.

Maslach, C. y Jackson, S.E. (1981). The measurement of experienced burnout. Journal of Occupational Behaviour, 2, 99-113.

Maslach, C., Schaufeli, W.B. y Leiter, M.P. (2001). Job burnout. Annual Review of Psychology, 52, 397-422.

Pines, A.M., (1993). Burnout: An existential perspective. En W.B. Schaufeli, C. Maslach y T. Marek (Eds.), Professional burnout: Recent developments in theory and research (pp. 33-52). Washington, DC: Taylor \& Francis.

Pines, A.M. y Aronson, E. (1988). Career burnout: Causes and cures. New York: Free press.

Schaufeli, W.B. y Bakker, A. (2007).
Burnout en bevlogenheid [Burnout y entusiasmo]. En W.B. Schaufeli y A. Bakker (Eds.), De psychologie van arbeid en gezondheid [Psicología del trabajo y la salud] (pp. 341-358). Houten: Bohn Stafleu Van Loghum.

Schaufeli, W. y Dierendonck, D. van. (2000). Utrechtse Burnout Schaal, Handleiding [la Escala del Burnout de Utrecht. Manual]. Lisse: Swets y Zeitlinger B.V.

Schaufeli, W. y Enzmann, D. (1998). The burnout companion to study y practice: A critical analysis. London: Taylor \& Francis.

Sherer, M., Maddux, J.E., Mercandante, B., Prentice-Dunn, S., Jacobs, B. y Rogers, R.W. (1982). The self-efficacy scale: Construction and validation. Psychological Reports, 51, 663-671.

Shirom, A. (2003). Job-related burnout: A review. En J.C. Quick y L.E. Tetrick (Eds.), Handbook of Occupational Health Psychology (pp. 245-264). Washington, DC:American Psychological Association.

Smith, D.B. y Moen, P. (1998). Spousal influence on retirement: His, her, and their perceptions. Journal of Marriage and the Family, 60, 734-744.

Sundin, L., Hochwälder, J., Bildt, C. y Lisspers, J. (2007). The relationship between different work-related sources of social support and burnout among registered and assistant nurses in Sweden: A questionnaire survey. International Journal of Nursing Studies, 44, 758-769.

Taylor, M.A. y McFarlane Shore, L. (1995). Predictors of planned retirement 
Burnout e intenciones de jubilación anticipada entre empleados mayores

age: An application of Beehr's model. Psychology and Aging, 10, 76-83.

Visser, P., Henkens, K. y Schippers, J. (2003). Beeldvorming en stereotypering van oudere werknemers in organisaties [Creación de imágenes y estereotipos de los empleados mayores en las organizaciones]. Gedrag en Organisatie [Comportamiento y organizaciones], 16, 2-22.
Wapperom, L., Boskemper, M. y Schouten, E. (2006). Levensloop of spaarloon? [¿Ahorro laboral o ahorro fiscal?] ${ }^{2}$ Hoofddorp: Kluwer.

Winnubst, J. (1993). Organizational structure, social support, and burnout. En W.B. Schaufeli, C. Maslach y T. Marek (Eds.), Professional burnout: Recent developments in theory and research (pp. 151162). Washington, DC: Taylor \& Francis. 